\title{
Relaciones entre la investigación y la formación docente permanente: El conocimiento necesario para la diversidad*
}

\author{
Relations between Research and Continuing Education Teacher: \\ Knowledge Necessary to Diversity \\ Relações entre a pesquisa e a formação continuada do professor: \\ Saberes necessários à diversidade
}

\author{
Carla Helena Fernandes. ${ }^{a}$
}

${ }^{a}$ Universidade do Vale do Sapucaí, Brasil. Fono: 55-19-3386-9505 e 55-19-9246-9904.

Correo electrónico: carlahelenafernandes@yahoo.com.br

\begin{abstract}
RESUMEN
La universalización de la educación para todos los niños y jóvenes debe considerar la diversidad social y cultural y desarrollar prácticas pedagógicas adecuadas. Afirmamos la necesidad de repensar la formación del profesorado teniendo en cuenta tanto el conocimiento técnico como una reflexión sobre la rutina de la escuela. La investigación llevada a cabo por el profesor sobre su propio trabajo es fundamental para su formación profesional y para la redefinición de las prácticas de la enseñanza. Se presenta el resultado de una investigación realizada con maestros de Enseñanza Fundamental de una escuela de Campinas, en São Paulo, Brasil. La investigación, de enfoque cualitativo, hizo uso de entrevistas semi-estructuradas y análisis de los relatos escritos de los maestros. Los resultados indicaron los cambios una vez que la investigación fue facilitadora de reflexiones y de nuevos conocimientos acerca de los estudiantes.
\end{abstract}

Palabras clave: investigación, formación docente permanente, diversidad.

\begin{abstract}
The universalization of education for all children and young people should consider the social and cultural diversity and build appropriate pedagogical practices. We affirm the need to rethink teachers' training considering, both, technical knowledge as well as a reflection on the school routine. A survey taken by teachers about their work is critical to their professional training, and to the redefinition of teaching practices. Here is the result of a research developed with elementary school teachers from a school in Campinas, state of São Paulo, Brazil. The research, with qualitative approach, made use of semi-structured interviews and analysis of written accounts of teachers. The results guided to changes as the investigative processes helped to build reflections and new knowledge about students.
\end{abstract}

Key words: research, teachers' permanent education, diversity.

\section{RESUMO}

A universalização da educação para todas as crianças e jovens deve considerar a diversidade social e cultural e construir práticas pedagógicas apropriadas. Afirma-se a necessidade de se repensar a formação profissional docente considerando-se tanto os saberes técnicos como a reflexão sobre o cotidiano escolar. A pesquisa realizada pelo professor sobre seu próprio trabalho é fundamental para sua formação profissional e para a ressignificação das

La investigación fue apoyada y financiada por el CNPq - Consejo Nacional de Desarrollo Científico y Tecnológico de Brasil. 
práticas docentes. Apresenta-se resultado de pesquisa desenvolvida com professores do Ensino Fundamental de uma escola de Campinas, no Estado de São Paulo, Brasil. A pesquisa, de abordagem qualitativa, fez uso da entrevista semiestruturada e da análise dos relatos escritos dos professores. Resultados indicaram para mudanças uma vez que os processos investigativos foram propiciadores de reflexões e de novos conhecimentos acerca dos alunos.

Palavras chave: pesquisa, formação continuada docente, diversidade.

\section{INTRODUCCIÓN}

La educación es un derecho de todos garantizado en la "Declaración Mundial sobre Educación para Todos y Plan de Acción para Satisfacer las Necesidades Básicas de Aprendizaje" (UNESCO, 1990) y, específicamente en Brasil, en la Constitución Federal (Brasil, 1988), en la Ley 8.069 que instituyó el "Estatuto del Niño y del Adolescente" (Brasil, 1990), y en la Ley de Directrices y Bases de la Educación Nacional (Brasil, 1996). La garantía de ese derecho requiere la creación de escuelas de calidad que realmente atiendan la diversidad social y cultural de Brasil y, de forma general, toda América Latina.

En Brasil, el movimiento de democratización de la escuela pública ocurrido en los años veinte del siglo XX defendía que la escolarización se extendiese a toda la población brasileña y, gradualmente, tuvieran acceso a la escuela niños y jóvenes de grupos sociales que permanecían alejados de ese proceso. Recientemente, en ese país, a partir de la década de los 90, la llamada "inclusión social y escolar" también ha propiciado reflexiones acerca de las prácticas escolares y pedagógicas, indicando la necesidad de cambios para que todos tengan acceso, aprendizaje y permanencia en las escuelas.

En ese marco, el trabajo docente tiene un papel de importancia como agente de la inserción social y escolar de los alumnos venidos de diferentes grupos sociales. No obstante, entre los profesores hay un recurrente discurso de falta de preparación con la afirmación de considerarse no aptos para enseñar a alumnos con actuación y desempeños tan diversos, afirmando incluso que los conocimientos adquiridos en el curso de formación inicial se muestran inadecuados o insuficientes en el atendimiento a esa diversidad. En ese sentido, en la capacidad de la construcción de una escuela que realmente contemple la diversidad humana, social y cultural, propusimos pensar junto a los profesores sobre la formación profesional en el contexto actual, indicando que la base de esa formación debería ser la reflexión sobre la práctica y el establecimiento de puentes entre la cotidianidad escolar, el ejercicio docente y los conocimientos de la formación inicial. Planteamos, en este sentido, la adopción de una perspectiva de formación profesional que tiene en la investigación desarrollada por el profesor y con el profesor el espacio-tiempo de esa reflexión (Elliott, 1998; Duran, Saury y Veyrunes, 2005; Geraldi, Fiorentini y Pereira, 1998; Zeichner, 1998a, 1998b).

Si entendemos la formación continuada como el aprendizaje promovido en el ejercicio profesional y en la escuela (Canário, 1998; Nóvoa, 1995; Tardif, 2007), entonces las condiciones de trabajo del profesor y la realidad con la cual se enfrenta diariamente y que, en muchos momentos, acaban por direccionar el trabajo realizado, representan situaciones de formación profesional, así como lo son los cambios con los pares, alumnos y otros interlocutores, lo que es preciso considerar en nuestras reflexiones. $\mathrm{Si}$, además, añadimos a tales situaciones una reflexión sobre su dirección, podemos hablar de investigación del trabajo docente y de la relación de esa investigación con una formación profesional.

La reflexión que presentamos en este texto tiene como referencia, además de los autores anteriormente citados que se dedican a estudiar la formación profesional y la investigación 
en la formación, investigación desarrollada con profesores de Enseñanza Fundamental en una escuela pública del sistema municipal de enseñanza de la ciudad de Campinas, en São Paulo, Brasil. A partir de las dificultades encontradas para enseñar a un grupo de alumnos, esos profesores se propusieron reflexionar e investigar su propia práctica, individual y colectivamente, lo que propició mudanzas y mejoras significativas en la sala de aula y en la escuela. Esta investigación fue pautada, junto a los profesores, en torno a los procesos formativos por los cuales pasan mientras están inmersos en el mundo escolar y, para explicar mejor las relaciones que pudieron ser evidenciadas en nuestra permanencia en la escuela, hacemos uso de una metáfora: la de hilos y nosotros que, en tramas que dan origen, construyen un tejido. En ese caso, en la escuela son hilos profesores, alumnos e investigadores que componen su cotidiano, viven y trabajan en la construcción de la educación escolar efectivamente volcada en su función educativa y social.

\section{FORMACIÓN PROFESIONAL DE PROFESORES E INVESTIGACIÓN EDUCACIONAL: RESIGNIFICANDO CONOCIMIENTOS Y PRÁCTICAS}

A lo largo de la historia de la educación escolar, en las políticas públicas destinadas al área, en la legislación y documentos oficiales, y en los innumerables programas y acciones desarrolladas como consecuencia de esas políticas y leyes, el cuadro que históricamente se construyó de la formación profesional de profesores en Brasil fue el de: 1) cursos de formación inicial bajo la responsabilidad única de las universidades, y 2) programas y acciones de formación continuada promovidas por los sistemas de enseñanza (también colaborando con las universidades), formados, en su mayoría, por cursos de corta duración con sumario y programación que apuntaban a la adquisición, por parte de los profesores, de conocimientos que no siempre iban al encuentro de sus necesidades. En ambos casos -en la formación inicial y continuada- el lugar del profesor era el de quien recibe pasivamente un determinado conocimiento construido fuera de la escuela de Educación Básica.

Según Tardif (2007), los investigadores y los profesores componen dos grupos con funciones distintas. El primero, formado por los productores del saber/conocimiento; el segundo, por los ejecutores, lo que acabó en serias consecuencias para el profesor y su trabajo en función del distanciamiento y de las diferencias entre las concepciones y prácticas de esos dos grupos. Apenas recientemente se pasó a valorar los conocimientos producidos por los sujetos de la escuela y a valorizarse también el espacio escolar como locus privilegiado de la formación profesional de sus profesores, lo que aún está en construcción. Además de eso, la propia demanda de la escuela y de sus profesionales por la reflexión de las situaciones vividas en la escuela ha solicitado otras modalidades de formación, lo que en muchos casos ha sido incentivado y acompañado de acciones en cooperación con la universidad. En ese proceso, se fue construyendo una red de acciones apuntando a la escuela, a la sala de aula y a las prácticas pedagógicas, lo que ha alterado la propia idea de formación profesional de profesores, y la enseñanza y el aprendizaje han sido colocados realmente como objetos de la formación.

En ese sentido, reafirmamos que los conocimientos de la formación inicial deben ser resignificados en lo cotidiano del trabajo docente a partir de lo nuevo que atraviesa el conocimiento de los profesores, novedad que está presente en cada alumno que se adentra en la escuela provocando otras situaciones y acontecimientos. Al escuchar a este nuevo 
alumno el profesor podrá escuchar sus conocimientos culturales y sociales, y lo otro alumno y conocimiento- puede representar medios de reflexión y cambio para el profesor.

Con Denari (2004) defendemos que el conocimiento profesional del profesor viene de la relación entre teoría y práctica, del establecimiento de puentes entre esos conocimientos, lo que puede ser propiciado por el establecimiento de articulación entre la formación inicial y la formación continuada. De acuerdo a esta autora:

La práctica pedagógica profesional como fuente permanente y privilegiada de reflexión y de actuación propicia el análisis del complejo movimiento existente entre las construcciones teóricas y las actividades prácticas, promoviendo la comprensión de la naturaleza y de la especificidad del conocimiento, propiciando el desarrollo de un compromiso ético y político común a la sociedad democrática (2004: 70).

Es en ese sentido que debemos buscar construir formación profesional que propicie el acogimiento de las necesidades apremiantes y de la realidad concreta y actual de los alumnos en oposición a las concepciones y prácticas de formación basadas en verdades absolutas e inmutables. En esta dirección, hace cerca de dos décadas en Brasil se ha vuelto a las reflexiones y acciones que apuntan a la construcción de lo que ha sido llamado "educación para la diversidad". Esta propuesta se traduce en las políticas públicas educacionales (Brasil, 2008, 2007, 2003), donde la educación para la diversidad afirma la urgente necesidad de que la escuela contemple los conocimientos de los diferentes grupos sociales y culturas y, además de eso, propicie en su interior reflexión crítica acerca de las cuestiones sociales y económicas que rodean esos grupos, proponiendo la construcción de prácticas pedagógicas que van al encuentro de sus potencialidades y necesidades, apartándose de las concepciones y prácticas hegemónicas. Las raíces socioculturales están en la base de la constitución de los pueblos y naciones, en especial en los países latinoamericanos; esa base sociocultural precisa ser también la referencia de la organización de la escuela y de los currículos escolares.

Un mayor nivel de equidad en el contexto educacional implica avanzar en dirección a la creación de escuelas que promuevan la educación en la diversidad, entendida como recurso para la mejoría de la calidad educacional y fuente de enriquecimiento humano. Según el principio que guía el Marco de Acción de la Conferencia Mundial de Salamanca sobre Necesidades Educacionales Especiales: Acceso y Calidad (Salamanca, 1994), todas las escuelas deben acoger a todos los niños, independientemente de sus condiciones personales, culturales o sociales -niños con deficiencia, niños de la calle, de minorías étnicas, lingüísticas o culturales, de áreas desfavorecidas o marginales- lo que constituye un importante desafío para los sistemas escolares. Para que las escuelas tengan condiciones de enfrentar este desafío, todos los profesionales del área educacional y, en especial, los docentes precisan contar con ayuda y apoyo permanente que posibiliten la participación continua en actividades de formación así como de asesoramiento al desarrollo de la práctica docente (Duk, 2005:12-13).

En el seno de esa discusión que solicita otra perspectiva de educación, están siendo construidas posibilidades de enseñar para todos los niños, lo que para los profesores representa un desafío a ser conquistado, solicitando formación centrada en la práctica, en 
la realidad y en el ejercicio docente, siendo el alumno concreto, y no un alumno idealizado, el foco de los procesos de enseñar y aprender.

Aunque esa demanda de escolarización sea amplia y solicite una actuación profesional competente y adecuada, para Fonseca "no es preciso inventar nuevos profesores, ni nuevos especialistas; es preciso, sí, valorar los profesores ya existentes con medidas adecuadas" (1995: 224), lo que implica, añadimos, la valorización de su trabajo. Para este autor, quien refuerza lo que venimos discutiendo, las dudas y reflexiones del profesor deben ser remitidas a la propia formación y, en ese campo, la investigación comprendida como la reflexión sobre la práctica docente a partir de los choques de lo cotidiano y del ejercicio profesional, puede constituirse en un importante instrumento de su formación profesional.

Sin embargo, la pregunta que hacemos y que también ha sido hecha por investigadores de las universidades y de las escuelas de Educación Básica es: ¿cómo relacionar la investigación y la formación del profesor? Comprendemos que rescatar la historia de la investigación como investigación de la práctica docente podrá llevarnos a las respuestas.

Como ya venimos afirmando, en Brasil la formación profesional docente se construyó y se consolidó teniendo como referencia el paradigma de la racionalidad técnica basado en la disociación teoría-práctica y en la idea de "práctica" como simple aplicación de la teoría, pero tal paradigma se mostró inadecuado a la luz de la complejidad de la cotidianidad escolar y de la construcción de conocimientos necesarios para el profesor inmerso en tal contexto, llevando a la crisis y al surgimiento de otro paradigma cuya base es la epistemología de la práctica (Cruz, 2003), esto es, situaciones prácticas a ser tratadas en toda su complejidad para que dentro de ellas se produzca conocimiento válido que permita a los sujetos actuar de forma constructiva. En ese nuevo paradigma, la formación profesional tiene como concepto la práctica docente y el aprendizaje del alumno real. A partir de dicho paradigma de investigación, se desarrollaron estudios que fueron poniendo foco en la relación entre investigación, docencia, escuela/universidad y formación.

Stenhouse (1975) fue uno de los primeros investigadores que se ocupó de la investigación en relación con la docencia en todos los niveles de enseñanza, en las universidades y escuelas, indicando la importancia del profesor-investigador. Stenhouse creó el CARE -Center for Applied Research in Education-, en la Universidad de East Anglia, en la Inglaterra de los años sesenta. Elliott (1998), uno de los seguidores de las ideas de Stenhouse, acrecentó aquellos estudios e investigaciones iniciales con otros estudios sobre modalidades de investigación que relacionaron acciones de los investigadores en la escuela junto a los profesores, y a esa idea Elliott la llamó investigación-acción. En la década de los 90, Zeichner (1998b) afirma la investigación como acción y reflexión crítica del profesor acerca del trabajo docente, en su relación con el contexto escolar y social. La reflexión para este autor debe tener como foco ese posicionamiento crítico generador de mudanzas. En común, esas investigaciones indicaban diferentes formas de pesquisa centradas en el contexto del trabajo, y con la participación de investigadores de la escuela o de la universidad.

Esas modalidades de investigación, en su relación con la formación del profesor, son especialmente provechosas y adecuadas para la construcción de educación escolar para la diversidad en el sentido de que lo nuevo (alumno, currículo, situaciones de enseñanza) es disparador de la propia investigación a partir de las necesidades levantadas por los sujetos partícipes. Cuando se trata de cuestionamientos acerca de prácticas pedagógicas y docentes encaminadas a la diversidad, la idea de un profesor que investiga su propia práctica va al encuentro de las necesidades y mudanzas a ser realizadas. 
Hace cerca de una década han sido desarrolladas prácticas cooperativas de investigación (Fiorentini, 2004) entre profesores e investigadores de las escuelas de educación básica y de las universidades, lo que exige que profesor e investigador se reconozcan mutuamente como productores de conocimiento, reconociendo sus saberes y experiencias. La relación entre investigadores y profesores puede ser movilizadora de construcciones compartidas y de la resignificación de aquello que inicialmente conocían, de manera tal que ambos profesionales pueden convertirse en investigadores. En esa construcción compartida, la interlocución entre investigadores de la universidad y de la escuela es constitutiva de esos profesionales y de sus conocimientos. Los sentidos son producidos en la interlocución yo-otro establecida entre diferentes sujetos que actúan en la escuela como un colectivo. Se rescata aquí la importancia del profesor-investigador (Stenhouse, 1975) en la contemporaneidad de las discusiones que ocurren en la escuela y sobre la escuela.

\section{LA INVESTIGACIÓN EN LA ESCUELA: ACERCA DE LOS CURSOS DE EDUCACIÓN CONTINUADA Y PRÁCTICAS COTIDIANAS}

La investigación a la que hacemos referencia en este texto fue desarrollada en una escuela pública del sistema municipal de la ciudad de Campinas, en el Estado de São Paulo, Brasil, con el objetivo de investigar junto a profesores de Enseñanza Fundamental los procesos formativos que acontecen en la escuela y que tenían como foco las prácticas pedagógicas realizadas por ellos. Fueron sujetos de esa investigación once profesores de Enseñanza Fundamental, estando entre ellos dos profesoras que actuaban directamente en el atendimiento y orientación de alumnos con necesidades educacionales especiales, nombradas en aquella red de enseñanza como "profesor(a) de Educación Especial".

La escuela, en la época del desarrollo de la investigación, atendía cerca de quinientos alumnos ofreciendo Enseñanza Fundamental en tres turnos de funcionamiento: matutino, intermedio y nocturno. El barrio donde está localizada es formado por familias de trabajadores de comercio y de industrias de la región de Campinas, muchos de los cuales estaban en desempleo. De los datos de aquel periodo, extraemos que, al final de la Enseñanza Fundamental, la expectativa de los alumnos y familiares era que los mismos concluyesen la Enseñanza Media aspirando a mejores condiciones de vida y trabajo. Entretanto, en la época, muchos alumnos salían de la Enseñanza Fundamental buscando un trabajo que les posibilitase ayudar a la familia, lo que los llevaba a la transferencia para el periodo nocturno y, en algunos casos, al abandono de los estudios.

En el universo escolar, locus de la investigación, fueron tomados como espacios de investigación: a) el acompañamiento individual de los profesores en sus clases y/u otras formas de atendimiento a los alumnos, y b) las reuniones pedagógicas realizadas entre los profesores y el equipo de gestión de la escuela. En esa investigación, fueron empleadas entrevistas semi-estructuradas y, con la aprobación de los profesores, fue realizado el análisis de los textos que los mismos docentes presentaron a la investigadora, textos que presentaban reflexiones escritas acerca de su trabajo.

En aquel municipio los(as) profesores(as) de Educación Especial asumen diferentes modalidades de atendimiento a los alumnos que presentan necesidades educacionales especiales, a saber: en la escuela y en la sala de aula, en salas de recursos multifuncionales, en clase hospitalar y en el atendimiento domiciliar. Los(as) profesionales deben tener formación específica, actúan directamente con los alumnos y participan de las demás actividades, de las cuales también participan los otros profesores. 
La escuela es viva y su cotidiano está marcado por la complejidad y por las contradicciones producidas entre lo micro y lo macro, entre los sujetos y las políticas públicas educacionales, entre las familias, la comunidad y el propio sistema de enseñanza municipal. En el periodo en que la investigación fue desarrollada, la escuela reflejaba en su interior, por un lado, 1) propuestas y orientaciones de la red municipal de enseñanza de Campinas - SP, que apuntaban a mudanzas curriculares, organizacionales, administrativas y profesionales ${ }^{2}$ que serían decisivas para la escuela y sus profesores en los años venideros y, por otro lado, y relacionado directamente con el aprendizaje de los alumnos, 2) reflejaba las inquietudes de los profesores acerca de las dificultades en la interacción con los educandos, lo que se extendía a dificultades para enseñar. Esas dificultades eran identificadas por los profesores como la no aceptación de las reglas escolares por los alumnos, y llamadas por los profesores como "indisciplina", habiendo en esa época una queja casi generalizada de los profesores acerca de la indisciplina de los alumnos del sexto al noveno año. Los profesores afirmaban que no conseguían enseñar y que los alumnos no aprendían pero, al escuchar a esos alumnos, oíamos sobre las dificultades de interacción con los profesores que, según esos alumnos, no valoraban su realidad y necesidades.

Esas dificultades presentes en la cotidianidad escolar promovieron la búsqueda de respuestas por los profesores y, en ese sentido, el contacto con una universidad pública de la región y sus investigadores promovió el desarrollo en la escuela de diferentes investigaciones desarrolladas por profesores de la propia escuela, investigadores de la universidad y por ambos, en proyectos y acciones investigativas y colaborativas que apuntaban a la mejoría de la escuela y del trabajo docente. Entre esas investigaciones, entre los años 2003 y 2008, fue desarrollado en la escuela un proyecto colectivo de estudios e investigación, el Proyecto "Escuela Singular: Acciones Plurales", del cual participaron casi la totalidad de profesores que, como investigadores becarios de una agencia de fomento del Estado de São Paulo, reflexionaron e investigaron sobre su trabajo.

En función de los objetivos de la investigación desarrollada por esta investigadora y presentada aquí -"Pareceres de profesores de la/en la formación continuada: Posibilidades de autoría (2009)"-, a saber, investigar los procesos formativos en la escuela, más allá de las acciones propias de la investigación, fueron también tomadas para su observación algunas de las acciones del Proyecto "Escuela Singular: Acciones Plurales", por ejemplo: 1) el acompañamiento por parte de esta investigadora del grupo de estudio (el llamado GTzinho)

1) Mudanzas curriculares promovidas por la implantación a nivel nacional de la llamada "Escuela de Nueve Años", con la matrícula de alumnos de seis años en el primer año de la Enseñanza Fundamental a partir de 2006, y por la implantación de la Enseñanza Fundamental en ciclos de tres fases (y tres años) a partir de 2006. 2) Mudanzas organizacionales para toda la red municipal, no sólo por la implantación de la Escuela de Nueve Años, sino también por la pretendida restructuración de las escuelas de aquella red municipal. En el caso de esa escuela, la Secretaría Municipal pretendía (era ésta una de las propuestas hechas por la Secretaría al colectivo de la escuela) que a partir de 2006 la escuela mantuviese apenas el primer y el segundo ciclo, con la ampliación del número de clases de cada año. 3) Mudanzas administrativas y profesionales como la propuesta de mudanzas en el Plano de Cargos, Carreras y Vencimientos del Magisterio Público Municipal de Campinas que se refería, entre otros aspectos, a la función profesional de los profesores, trabajo docente colectivo, formación profesional continuada, jornada de trabajo, remuneración y carga horaria.

3 Coordinaron el Proyecto "Escuela Singular: Acciones Plurales" profesores de la Facultad de Educación de UNICAMP. El Proyecto fue registrado junto a FAPESP -Fundación de Apoyo a la Investigación del Estado de São Paulo-, órgano de fomento a la investigación. 
organizado por los profesores, y 2) la lectura analítica de los informes de investigación ${ }^{4}$ de los profesores participantes del referido proyecto, cuya redacción fue acompañada por esta investigadora. Entendiendo el texto como un medio de reflexión de los docentes (Prado y Fernandes, 2009, 2008; Prado y Soligo, 2005) comprendemos la elaboración de esos informes como una posibilidad de comprensión de los problemas y dilemas puestos en práctica por los profesores.

\subsection{DILEMAS PUESTOS EN PRÁCTICA: INVESTIGACIÓN Y FORMACIÓN}

Como afirmamos, en la perspectiva de la investigación como espacio de formación, lo que moviliza a profesores e investigadores son los dilemas puestos en práctica, el ejercicio cotidiano y los conocimientos de la formación inicial. En el contexto de la escuela, la gradual inclusión de alumnos de grupos sociales y culturales que históricamente permanecían fuera de la educación formal promovió, y ha promovido, la desestabilización de certezas de los profesionales y de saberes instituidos, como afirma Tardif (2007). La idea de "dilema" tiene como referencia los estudios de Pacheco, quien lo entiende como sigue:

Lo que los profesores saben se define como un cuerpo de significados, conscientes o inconscientes, que surgen a partir de la experiencia y que se expresan en acciones personales. Es un conocimiento íntimo, social y tradicional que se orienta más para la acción y se contextualiza por la profesión. En este campo, se estudian igualmente las situaciones problemáticas de los profesores, que generalmente se simbolizan como dilemas. Un dilema se define por una situación problemática que los profesores enfrentan sea a nivel de pensamiento, sea a nivel de la práctica, en el discurso de la actividad profesional, surgiendo como un punto de tensión que el profesional resuelve y a partir del cual toma decisiones (1995: 55).

De los once profesores que participaron en la investigación, presentamos en la secuencia extractos de las producciones de cuatro, estando entre ellos la profesional que actúa directamente con los alumnos con necesidades educacionales especiales matriculados en la escuela. Los extractos indican la relación entre el surgimiento de la cotidianidad escolar y las reflexiones llevadas a cabo por los docentes (individual y colectivamente), comprendiendo esas relaciones como parte del proceso de formación de los profesores.

En la presentación que sigue, los extractos presentados hablan sobre dos puntos/ aspectos de reflexión, a saber: 1) la relación entre la investigación y la formación, y; 2) dilemas promotores de reflexión e investigación. Su presentación objetiva busca resaltar, en los pareceres de los profesores, en este caso, plasmados en los informes de investigación, las inquietudes que fueron movilizadoras de reflexiones y mudanzas, resaltando también la presencia de lo otro (alumno y profesor) en las reflexiones de ese profesor.

Fueron presentados por los profesores textos producidos por los mismos en diferentes géneros, pero los informes de investigación presentados al Proyecto "Escuela Singular: Acciones Plurales" fueron seleccionados como objetos de investigación en función de lo que a la época representaban para los profesores, a saber, su inserción al mundo de la investigación y la materialización de sus reflexiones. En su análisis, fue considerado por la investigadora el origen de ese género en la universidad y el hecho de que, en función de ese origen, cargan marcas de un texto propio a aquel contexto y ajeno a la escuela (ese análisis fue presentado en Fernandes, 2009). 


\subsubsection{Investigación y formación}

En los extractos que siguen se observa, como venimos afirmando, que los dilemas vividos por los profesores fueron disparadores de sus búsquedas e investigaciones:

[IIM15]: Ese proyecto surgió de una necesidad de la propia escuela, en un movimiento de búsqueda de soluciones para los dilemas a los que nos enfrentamos diariamente. Por este motivo, el equipo de gestión (directora, vice-directora y orientadora pedagógica) también participa de él, lo que facilita bastante el curso del trabajo. Estando todos inmersos (incluso sabiendo que hay diferentes niveles de implicación y de creencia), las necesidades son sentidas por todos y hay mayor facilidad en comprender las prioridades y las necesidades del grupo.

[IIR1]: Este proyecto tuvo inicio con la búsqueda de solución al problema de indisciplina que sufría todo el cuerpo docente y consideré importante mi participación visto que tenía grandes problemas de indisciplina en la sala de aula. Además de eso, vi el proyecto como una oportunidad de estudiar esta temática e intentar comprender la cuestión, intercambiar experiencias con los colegas y así llegar a una solución.

[IIMA1]: En 2004, nuevos miembros se integraron al grupo de estudio, un proyecto más amplio nos trajo la oportunidad de pensamiento y acción sobre la realidad, las expectativas anteriores fueron atenuadas. La constitución del nuevo grupo fue un proceso de aproximación, seducción y confianza, la conquista del espacio de un trabajo de equipo, los coordinadores del grupo con sus orientaciones a nuestros dilemas pedagógicos, en los esclarecimientos de temas estudiados como la indisciplina, la evaluación, los procesos de enseñanza-aprendizaje, la motivación, reflejados y discutidos con diferentes visiones, fueron compañeros que incentivaron la práctica profesional pensada en una perspectiva de mudanza y diálogo.

Los extractos focalizan la movilización de los profesores a la investigación, aspirando la superación de los dilemas. La investigación, en este sentido, era comprendida como espacio de formación a partir de las reflexiones y discusiones colectivas propiciadas en la escuela, centrándose en la cotidianidad escolar, la práctica profesional y el ejercicio docente.

[IIR2]: Las lecturas tenían un solo objetivo: conocer las ideas del autor, confrontarlas, si es posible, con otras ya conocidas y llegar a conclusiones que me ayudasen a mejorar la interacción con mis alumnos y mi aula, intentando alcanzar al mayor número de alumnos, despertando su interés por la Matemática. Buscar resolver los problemas de aprendizaje y de desempeño identificados en la Matemática, y lo que alcanza un gran número de nuestros alumnos también era nuestro objetivo. Vencer aquellas viejas reticencias de los alumnos -"No me gustan las Matemáticas"- y de las escuelas -"Matemática es el área que más suspende"- también hacían parte de las preocupaciones. Realmente estoy en la búsqueda de una fórmula para resolver estos problemas que me acompañan como profesor de Matemáticas.

Los extractos son identificados por las iniciales II "Informe de Investigación”, seguido de la inicial del nombre de cada uno de los cuatro profesores (R, M, A y MA) y el número de los pasajes correspondientes. 
Específicamente en cuanto a la profesora que, en la escuela, atendía directamente a los alumnos que presentaban necesidades educacionales especiales, observamos que los dilemas se refieren a su contribución en el proceso enseñanza-aprendizaje como un todo, indicando su inserción en las discusiones y reflexiones sobre la escuela y sobre la inclusión. La búsqueda de esa profesora es también por el establecimiento de asociaciones necesarias.

[IIA1]: A partir de los dilemas y problemas vividos por nosotros en lo cotidiano de una escuela inclusiva y la posibilidad de la relación con la universidad, iniciamos un nuevo momento de trabajo, con orientación para investigaciones, estudios y descubrimientos. Tenemos el objetivo de, juntos, mejorar el proceso enseñanza-aprendizaje, invirtiendo en nuestro desarrollo profesional. Entre las categorías de objeto de estudio de nuestro proyecto, enfocamos nuestra atención en las acciones didáctico-pedagógicas e intentamos buscar, a través de nuestros estudios y reflexiones, cuáles decisiones y acciones deben ser tomadas dentro y fuera de la sala de aula, buscando la inclusión de alumnos con necesidades educacionales especiales.

\subsubsection{Dilemas de la práctica docente: Reflexión e investigación}

Como venimos afirmando, el cotidiano escolar y los procesos de enseñar movilizaban a los profesores a buscar, individual y colectivamente, soluciones para los enfrentamientos que se presentaban. En los informes, la escritura formal e impersonal de ese género discursivo ${ }^{6}$ fue siendo sustituida por la narrativa de la práctica docente, apropiada por el profesor como camino para la expresión y la implicación del autor con su propia experiencia, como se lee:

[IIR3]: Tengo como experiencia propia el caso de un alumno que en el quinto año no realizaba las actividades, teniendo siempre que llamar su atención para que comenzase a hacer y terminar, pues siempre paraba. Muchas veces, mientras gran parte de la sala había terminado, él estaba allí parado; algunas veces conversando o jugando con el colega. -"Profesor, ¡no me deja tranquilo!"- pasó a responder. Obtenía solamente desempeño insatisfactorio y si dependiese solamente de mi parecer no sería aprobado. Resolví, entonces, convocarlo dispensando a los otros alumnos que atendía. Quería acompañarlo individualmente para ver si identificaba algún problema. Como era víspera de examen, resolví simplemente estudiar junto a él la materia y mostrar que yo solamente quería ayudarlo. Hacía lo que yo proponía y hasta preguntaba lo que no entendía, cosa que nunca hizo en sala de aula. Durante la prueba, en el día siguiente, preguntó solamente si era igual a lo que habíamos estudiado juntos. Claro que si, respondí. Obtuve óptimo desempeño y el resto del año no tuve más problemas. Él pasó a hacer todas las actividades y se tornó atento cuando lo llamaba. Probablemente haya otros factores que influenciaron este cambio, pero es admirable cómo a partir de un simple refuerzo en la relación con el alumno, en la afectividad, se obtiene un resultado tan positivo en el aprendizaje.

Las reflexiones que ocurrían en los colectivos escolares, ya instituidos por la escuela o creados a partir del Proyecto "Escuela Singular: Acciones Plurales", se extendían a las reflexiones individuales presentadas en los textos de los profesores. Aspectos fundamentales,

Sobre este asunto ver "Los géneros discursivos" de M. Bakhtin ([1953] 2003). 
de base, de los procesos de enseñar fueron resignificados por los profesores teniendo como referencia esas reflexiones. En la investigación de la práctica, que incluía la sistematización de lo vivido, fue fundamental para esas resignificaciones y mudanzas.

[IIR4]: Teniendo como objetivo del proyecto el estudio y las reflexiones que llevasen consigo los cambios en la sala de aula para mejorar el proceso de enseñanza-aprendizaje, mudanzas basadas en fundamentos teóricos y/o experiencias vividas o compartidas, significando muchas veces una rotura de creencias y mitos que se arrastran hace siglos en la educación, tuvimos claridad de la forma como pienso y veo a los alumnos. Primero una creencia: creía que después de trabajar cierto contenido con los alumnos, aplicaría una prueba individual para verificar si el alumno aprendió o no, cuyo parámetro utilizado sería el resultado de la prueba, que suponía estar directamente relacionado al aprendizaje. Tan simple: haría, de esta forma, una evaluación del conocimiento de los alumnos. Basado en la lectura de los textos y en la discusión en el grupo de estudios pude concluir realmente que era una creencia.

Para la profesora que atendía alumnos con necesidades educacionales especiales, los dilemas que movilizaban su búsqueda se referían a la actuación propia, cuestionando sobre cómo dar atención al alumno y cómo desenvolver su trabajo en colaboración con los demás colegas. Los cuestionamientos de la profesora se refieren a su actuación en el cotidiano de la escuela y de la sala de aula, donde se incluían interrogantes sobre cómo debe ser desarrollado, por la escuela y los profesores, el proceso de escolarización de esos alumnos.

[IIA2]: Inicialmente presentamos los dilemas que hemos enfrentado en nuestra cotidianidad: ¿la actuación con el alumno deberá acontecer para que él sea realmente beneficiado? ¿Cuándo actuar? ¿Cómo actuar?, ¿dentro de la sala de aula o individualmente? ¿Cómo no perjudicar la rutina? Fuera de la sala, ¿estaría excluyendo? ¿Debo esperar al profesor para pedir ayuda? Y... ¿cuando no pide? [...] Lo que en un primer momento de conversación pude percibir es que lo que ha hecho el profesor, en la mayor parte de los casos, es pedir orientación del profesor de Educación Especial cuando el alumno no produce como espera el profesor. ¿Será que todos aquellos alumnos que no producen como espera el profesor necesitan realmente de una atención especial o es la manera como acontece el proceso enseñanza-aprendizaje para aquel alumno que es diferente de lo que se espera?

En otro párrafo del informe, la misma profesora resalta la necesidad de reflexión crítica y compartida, colectiva, en la superación de problemas y en el desarrollo de la práctica docente. Específicamente en el extracto inferior, la profesora anuncia la importancia de los colectivos de profesores.

[IIA3]: Para pensar en una escuela abierta a la diversidad, creo que hay necesidad de pensar en qué medida hemos favorecido el aprendizaje de todos los alumnos considerando la pluralidad dentro de la sala de aula. [...] Como también es necesario que el profesor entienda que sus alumnos desarrollen medios diferentes de aprendizaje y así utilizan caminos que el profesor puede desconocer. Percibo que para algunos profesores, el alumno que presenta una necesidad educacional especial, con deficiencia o no, es responsabilidad única del profesor de Educación Especial ya que, según ellos, no fueron formados para eso. Pero he 
percibido en este año que esta resistencia de los profesores ha tomado nuevos rumbos, teniendo en cuenta que un grupo de profesores se ha dedicado a alumnos con necesidades especiales en sus proyectos.

En el testimonio de otros profesores, las reflexiones y acciones colectivas entre docentes y alumnos se construyen en la complejidad de la cotidianidad escolar y son dependientes, o participan de las relaciones e interlocuciones establecidas.

[IIM2]: En este primer semestre algunos factores acabaron dificultando la concretización de una práctica interdisciplinar más efectiva. El primero de ellos, ya mencionado anteriormente, habla respecto al cambio de los profesores sustitutos, lo que acabó acarreando grandes cambios en la constitución de nuestro subgrupo. Precisamos, entonces, reiniciar todo ese trabajo de creación de vínculos con los nuevos profesores que pasaron a integrar el grupo. Además del establecimiento de vínculos entre nosotros como docentes, dentro del grupo existe también el desafío de establecer vínculos entre los nuevos profesores y los alumnos, que muchas veces los "testan" mucho en el primer momento, lo que dificulta el trabajo en clase. En varios momentos de nuestras reuniones, aspectos referentes a la relación profesor/alumno tomaron cuenta de las mismas dificultades, instancias que se acabaron tornando espacio de quejas y desahogos en relación a los problemas vividos dentro del aula.

La relación individual-social en la escuela y en la construcción del hacer docente es explicitada en el extracto anterior. Las profesoras indican a la escuela como espaciotiempo de interlocuciones importantes para el reconocimiento mutuo de los sujetos que allí conviven, posibilitando sustituir el individualismo por acciones colectivas.

[IIMA2]: Me encuentro aquí, tal como un personaje en un caminar, adecuándose a estas múltiples alteraciones. Nosotros que somos los sujetos, los alumnos, los padres, los funcionarios, los profesores, el equipo de gestión, ex-alumnos, ex-funcionarios, la comunidad escolar, haciendo el espacio para tener vida, en las relaciones de encuentros, desencuentros, idas y venidas alteradas anualmente, modificaciones necesarias que dejan sus marcas. Son las marcas de las vivencias y vínculos personales que nos enriquecen en nuestra formación y constitución de lo que somos, la imagen, la autoestima, en los valores éticos y morales, en fin, en nuestro cuerpo y en el alma. Esto se establece con la identificación con el otro, la afectividad, en la amistad, reconociéndome parte de un espacio y para el grupo.

\section{CONSIDERACIONES FINALES}

En la Introducción hicimos referencia a la metáfora de los hilos y tramas como representativa de lo que aquí presentaríamos. Promoviendo un rescate de los testimonios de los profesores sujetos de la pesquisa, lo que expusimos tuvo como objetivo evidenciar el surgimiento de los dilemas de los profesores y una movilización reflexiva y promotora de interlocuciones y mudanzas. En los testimonios de los profesores presentados en extractos de sus informes de investigación, se evidenció el nacimiento de situaciones comunes a los docentes porque fueron localizadas en la escuela. El término "nosotros" empleado por los profesores puede ser pensado también en el sentido de hilos que entrelazados producen 
diálogos y tramas, pudiendo afirmar la evidencia de un "nosotros" colectivo constituido en la interlocución, en las reflexiones y en las mudanzas necesarias.

En los testimonios de los profesores, un primer nudo evidenciado (y que construye la trama, en los amarres de los muchos hilos) es la relación entre las interlocuciones que se establecieron y el contexto de la escuela y las aulas, relación propiciada por las investigaciones desarrolladas por los profesores en la escuela, que tomaron como objeto de investigación aspectos y situaciones consideradas críticas por ellos mismos. Un segundo nudo se refiere a las relaciones establecidas entre profesores y el cruce de sus conocimientos con otros, conocimientos y sujetos, lo que, así concluimos, enriqueció su hacer y abrió otras/nuevas posibilidades de enseñar y aprender.

En las tramas que son producidas en la escuela se inserta, y lo queremos dar a conocer, el lugar del profesor que, en los entresijos de la misma y en el encuentro con otros profesores, construye su trabajo contribuyendo en la edificación de una escuela distinta, abierta a la diversidad, que contempla y respetuosamente enseña a todos los alumnos, teniendo como foco los dilemas puestos en la práctica, que en aquel momento de la escuela se caracterizaban por la "indisciplina" de los alumnos y por el bajo rendimiento escolar.

En ese sentido, reforzamos que las reflexiones presentadas en este texto tienen como punto central la interrelación constitutiva y constructiva entre la investigación y la formación profesional de profesores, cuyo eje, como hemos afirmado, son las prácticas docentes y pedagógicas en su continua resignificación.

\section{REFERENCIAS BIBLIOGRÁFICAS}

Bakhtin, M. ([1953] 2003). Os gêneros do discurso. Estética da criação verbal. São Paulo: Martins Fontes.

Brasil. (2008). Ministério da Educação. Secretaria de Educação Especial. Política Nacional de Educação Especial na Perspectiva da Educação Inclusiva. Brasília: MEC/SEESP.

(2007). Ministério do Planejamento, Orçamento e Gestão. Plano Plurianual 2008-2011.

Brasília: Ministério do Planejamento, Orçamento e Gestão. (2003). Ministério do Planejamento, Orçamento e Gestão. Plano Plurianual 2004-2007.

Brasília: Ministério do Planejamento, Orçamento e Gestão. (1996). Lei $n$. 9.394, de 20 de dezembro de 1996. Estabelece as diretrizes e bases da educação nacional. Brasília: Câmara dos Deputados.

(1990). Lei n. 8.069, de 13 de julho de 1990. Dispõe sobre o Estatuto da Criança e do Adolescente e dá outras providências. Brasília: Senado Federal.

. (1988). Constituição da República Federativa do Brasil. Brasília: Senado Federal. Secretaria Especial de Editoração e Publicações.

Canário, R. (1998). Gestão da escola: Como elaborar o plano de formação? Em R. Canário, Cadernos de Organização e Gestão Curricular (pp. 1-18). Lisboa: Instituto de Inovação Educacional.

Cruz, G. B. (2003). Pesquisa e formação docente: Apontamentos teóricos. Revista Eletrônica de Ciências da Educação, v. 2, n. 1. Recuperado de http://www.presidentekennedy.br/rece/trabalhosnum2/artigo04.pdf, consultado en febrero de 2013.

Duk, C. (2005). Educar na diversidade: Material de formação docente. Brasília: Ministério da Educação. Secretaria de Educação Especial.

Denari, F. E. (2004). Formação de professores em educação especial: A ótica do GTEE - 06 e do Fórum Paulista Permanente de Educação Especial. Em S. Omote (Org.), Inclusão: Intenção e realidade (pp. 61-76). Marília: FUNDEPE. 
Duran, M., Saury, J. e Veyrunes, P. (2009). Relações fecundas entre pesquisa e formação docente: Elementos para um programa. Cadernos de Pesquisa, vol. 35, n. 125, 37-62.

Elliott, J. (1998). Recolocando a pesquisa-ação em seu lugar original e próprio. Em C. Geraldi, D. Fiorentini e E. Pereira (Orgs.), Cartografias do trabalho docente: Professor(a)-pesquisador (a) (pp. 137-153). Campinas: Mercado das Letras/ALB.

Fernandes, C. H. (2009). Dizeres de professores da/na formação continuada: Possibilidades de autoria. Tese de Doutorado, Faculdade de Educação, Universidade Estadual de Campinas, Brasil.

Fiorentini, D. (2004). Pesquisar Práticas Colaborativas ou Pesquisar Colaborativamente? Em M. C. Borba e J. L. Araújo (Orgs.), Pesquisa qualitativa em educação matemática (pp. 47-76). Belo Horizonte: Autêntica.

Fonseca, V. (1995). Educação Especial: Programa de Estimulação Precoce. Uma introdução às ideias de Feuerstein. Porto Alegre: Artes Médicas.

Geraldi, C. M. G., Fiorentini, D. e Pereira, E. (Orgs.). (1998). Cartografias do trabalho docente: Professor(a)-pesquisador(a). Campinas: Mercado de Letras: ALB.

Nóvoa, A. (1995). Os professores e a sua formação. Lisboa: Dom Quixote.

Pacheco, J. A. (1995). O Pensamento e a aç̧ão do professor. Lisboa: Porto Editora.

Prado, G. V. T. e Fernandes, C. H. (2009). Saberes docentes tecidos na escrita: Pontos do fazer constituídos na autoria e na interlocução. Em M. P. Lacerda (Org.), A escrita inscrita na formação docente (pp. 64-82). Rio de Janeiro: Rovelle.

(2008). A narrativa na formação de professoras e de pesquisadores da/na escola: Diários de viagem. Educação Unisinos, vol. 12, n. 1, 16-27.

e Soligo, R. (2005). Porque escrever é fazer história: Revelações, subversões e superações. Campinas: Abaporu/UNICAMP.

Stenhouse, L. (1975). An Introduction to Curriculum Research and Development. London: Heinemann.

Tardif, M. (2007). Saberes docentes e formação profissional. Petrópolis: Vozes.

UNESCO. (1990). Declaração Mundial Sobre Educação Para Todos. Satisfação das Necessidades Básicas de Aprendizagem. Conferência Mundial sobre Educação para Todos. Brasília: UNESCO. Recuperado de http://unesdoc.unesco.org/images/0008/000862/086291por.pdf, consultado en febrero de 2013.

Zeichner, K. (1998a). Tendências da pesquisa sobre formação de professores nos Estados Unidos. Revista Brasileira de Educação, n. 9, 76-87.

(1998b). Para além da divisão entre professor-pesquisador e pesquisador-acadêmico.

Em C. Geraldi, D. Fiorentini e E. Pereira (Orgs.), Cartografias do trabalho docente: Professor(a)pesquisador(a) (pp. 207-236). Campinas: Mercado de Letras: ALB. 